\title{
Identification of Highly Jittered Radar Emitters Signals based on Fuzzy Classification
}

\author{
Yee Ming Chen, Chih-Min Lin, and Chi-Shun Hsueh \\ ${ }^{l}$ Dept. of Industrial Engineering and Management, Yuan Ze University, Chung-Li, Tao-Yuan, 320, Taiwan, \\ R.O.C. \\ ${ }^{2,3}$ Dept. of Electrical Engineering, Yuan Ze University, Chung-Li, Tao-Yuan, 320, Taiwan, R.O.C. ${ }^{2}$
}

\begin{abstract}
Emitter signals identification is one of the key procedures in signal processing of Electronic Intelligence (ELINT). Jitter is an unintentional form of modulation that can have a wide variety of sources. Timing-related data errors will occur if jitter is beyond acceptable limits. Designers need a fast and easy way to obtain a complete characterization of clock jitter in microprocessor controlled. To enhance the ability of emitter identification (EID) to meet the requirement of modern ELINT, a novel identification approach for radar emitter signals based on type-2 fuzzy classifier is presented in this paper. This work discusses the impact of unknown jitter sampling on signal estimation. Based on the ELINT feature extraction of radar emitter signals, the type-2 fuzzy classifier is applied to identification of highly jittered radar emitters effectively. Experiment results shows that the approach can achieve high accurate classification even at higher error deviation level, and has good characteristics of identification.
\end{abstract}

Keywords: - Signals identification, Fuzzy classifier, Electronic Intelligence

\section{INTRODUCTION}

Radar emitter signal identification is one of the key procedures of radar electronic intelligence (ELINT). In operational environments, it is important to know every radar emitter whether it is friend or foe, so that any counter measure could be applied in time. Number of homogenous pulse trains depends on radar transmitter power, sensitivity of ELINT equipment, distance to radar, antennas gain, methods of scanning and propagation conditions. Measurement information is included in so called pulse descriptor words (PDWs). Each PDW information depicting each particular pulse includes: radio frequency (RF), pulse amplitude (PA), pulse width (PW), pulse repetition interval (PRI), time of arrival (TOA), and direction-of-arrival (DOA) and so on. In the present days mostly, PDWs processing are virtually everywhere. The trend is toward more capable microprocessor-controlled systems that will run at faster and faster clock rates. As clock rates increase, characterization of timing accuracy and jitter becomes more important. Designers are finding an increased need to characterize jitter in order to achieve error-free design goals. Simply stated, jitter is just noise. The noise causes an uncertainty in the relative position of each clock cycle. Excessive clock jitter can limit performance in ELINT equipment. Because clock jitter is nothing more than the time variation between the edge of a clock signal and its ideal location in time, most engineers can intuitively relate to the basic definition of jitter. Period jitter is measured in the time domain and is expressed as either the rms or peak to peak of the time variation. Period jitter is defined as the variation in the time difference between the edge of a reference edge and the same edge of the clock uncertainty interval-valued clock cycles(Figure 1).

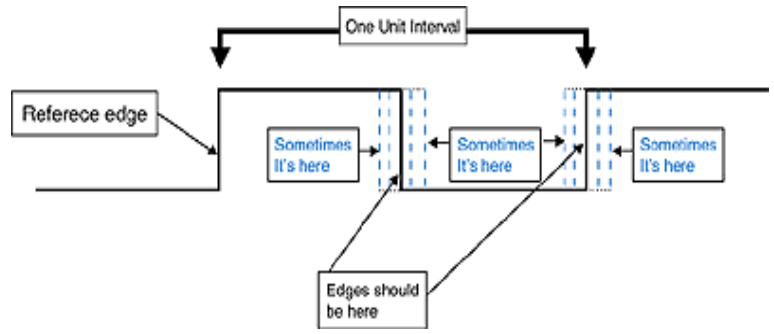

Figure 1. The clock uncertainty interval-valued clock cycles in ELINT modulation

Jitter is an unintentional form of modulation that can have a wide variety of sources. Timing-related data errors will occur if jitter is beyond acceptable limits. Designers need a fast and easy way to obtain a complete characterization of jitter in microprocessor controlled ELINT. The impact of jitter can vary widely from one application to another. Because jitter takes many forms, it is important for the engineer to understand 
which jitter measurement and emitter identification (EID) problems are most appropriate for ELINT equipment [1]. For many practical EID methods, including statistical pattern matching and probabilistic models [2, 3], data clustering [4], fuzzy classification [5] and neural networks [6-8] have drawn much attention and been applied in recent years. The statistical pattern recognition methods usually explore statistical properties in the data set and they perform well when such knowledge is known a priori. These methods are inefficient and time-consuming for solving EID problems. All these often fail to identify signals under high signal density environment, in near real time. Main problems arise from the uncertainty measures which may all decrease the accuracy of classification. Therefore, the primary focus of this paper is on the interpretability and uncertainty of PDWs. Fuzzy logic (FL) has proven its potential for estimating and minimizing the effect of uncertainties that are present in PDWs modeling environment [9]. This property plays an important role in this work aimed at handling the EID uncertainty dynamics in the given feature space. The type-2 (T2) FL is an emerging methodology that is capable of dealing with more than static imprecision in data [10]. Probability theory is used to handle random uncertainty and fuzzy sets are used to handle linguistic uncertainty, and sometimes fuzzy sets can also be used to handle both kinds of uncertainty, because a FL-based approach may use noisy measurements or operate under random disturbances. A type-2 fuzzy set has a capability to provide proper estimation of dispersion in uncertain conditions [11]. It is found that a type-2 fuzzy set is capable to handle and minimize the effect of both linguistic and random uncertainties, simultaneously. Hence, it is expected that a T2 fuzzy classifier should explicitly define the rule that governs an effective EID classification. In this work the concept of an application of a fuzzy classifier designed in the framework of type-2 fuzzy logic to the EID classification problem is developed. According to above information and due to existing uncertainty in EID problem, the design of a type-2 fuzzy classifier and its performance are discussed in this paper. Finally, the tests for the robustness of the classifier in the presence of jitter were carried out.

Section II outlines the background employed in this work. The EID data and a feature extraction procedure are described first. Next, we describe the type- 2 fuzzy sets. In section III design of a type- 2 fuzzy classifier and its operation scheme are proposed. In section IV the experimental results are presented and discussed. Finally, the paper is concluded and a scope of further work is suggested in section V.

\section{PROBLEM STATEMENT}

Radars typically emit thousands of pulses every micro second and in most scenarios there are many types of radar operating simultaneously interleaving radar pulse trains. The main function of ELINT is the realtime identification of the radar type associated with each pulse train that is intercepted. Current approaches adopted in actual applications are that the incoming emitter signals are sorted into individual pulse trains, then the characterizations of the pulse trains are compared with a library of parametric descriptions, a list of likely radar types will be yielded (Fig.2). The ELINT receiver picks up the pulses emitted by surrounding radars in the environment and measures a sequence of pulse descriptor words (PDWs), where a PDW is a data stream may be partitioned into two data streams called What and Where. Here the What data stream consists of parameters that characterize the functional aspects of radar systems. Such parameters include radio frequency (RF), pulse width (PW) and pulse repetition interval PRI, which is directly useful for radar type identification. The data stream consists of context-specific parameters. This stream is defined by parameters, such as bearing ( $\mathrm{Brg}$ ) and pulse amplitude (PA), that indicate the status (e.g.,position) of emitters in the environment and is less useful than What data steam for radar type identification. So in this paper, we choose the RF, PRI and PW (What data stream) to compose a PDW.

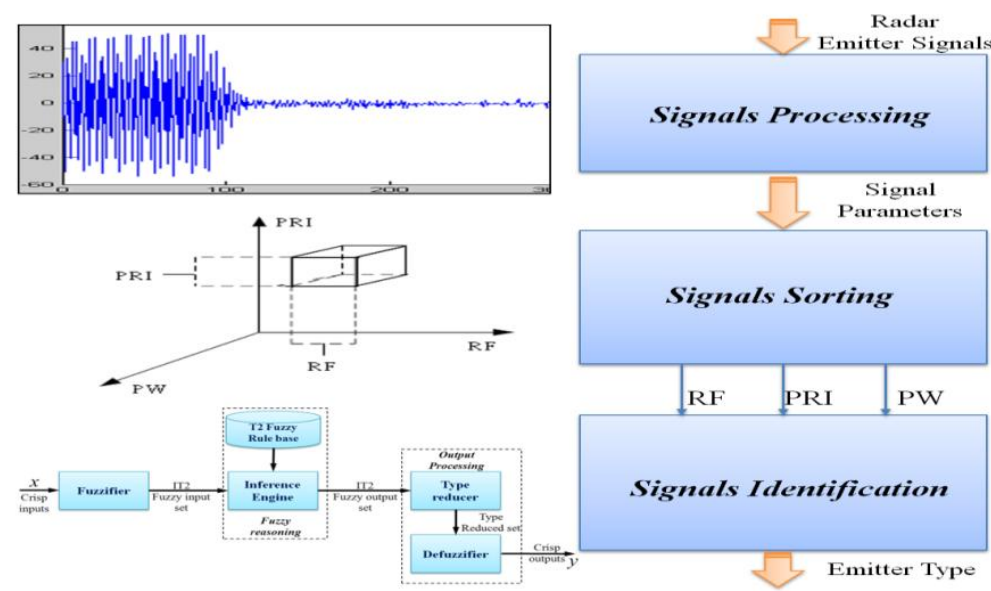

Figure 2. ELINT System for intercepting pulses used for this paper. 
The work presented in this paper is based on the EID real data recorded by a research group (named ABC Group) located in Taiwan. The pulse repletion interval (PRI) is in the range of $1.0 \mu \mathrm{s} \sim 10.0 \mu \mathrm{s}$, the pulse width (PW) is in the range of $0.1 \mu \mathrm{s} \sim 10.0 \mu \mathrm{s}$, the radio frequency of the carrier wave (RF) is in the range of $2.0 \mathrm{GHz} \sim 18.0 \mathrm{GHz}$. A sample of the radar pulse data set is described in Table 1.

The main difficulty is due to the capability limitations of ELINT sensors, which often fail to provide crisp value parameters and produce the range of parameter values for the emitters parameters, particularly emitters of complex characteristics. Interpretability and transparency of signal identification obtained with FLbased approach are also worth emphasizing. It is expected that a type-2 fuzzy classifier should explicitly define the rule that governs an effective radar emitter type classification. The details of the design method are described in following sections.

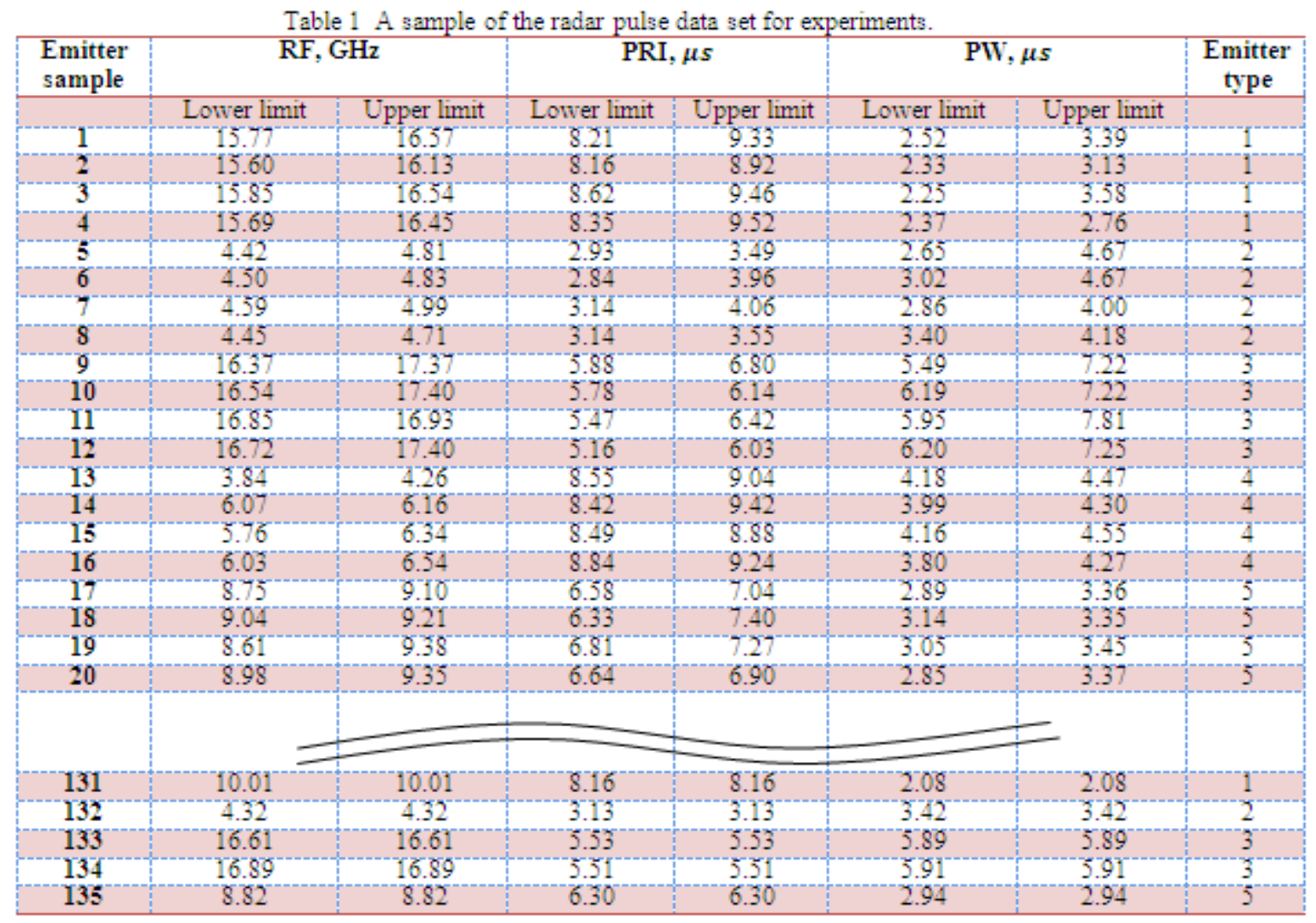

\section{TYPE-2 FUZZY CLASSIFIER DESIGN}

This research emphasis is placed on type-2 fuzzy methodology due to its capabilities to account for noisy measurements or operate under jitter disturbances associated with ELINT recordings. Type-2 fuzzy sets have been chosen to represent antecedents of fuzzy rules. A template of a fuzzy rule in the Mamdani framework is the following

$R^{n}:$ if $x_{1}$ is $\tilde{A}_{1}^{n}$ and $\cdots$ and $x_{i}$ is $\tilde{A}_{i}^{n}$ then $y$ is $Y_{j}^{n}$.

where $R^{n}$ is the nth rule, $n=1, \cdots N$, with each rule having $i$ antecedents, $\tilde{A}_{i}^{n}(i=1,2, \ldots, I)$ is the type-2 fuzzy membership functions of input variable. $Y_{j}^{n}=\left[y_{j}^{n}, \bar{y}_{j}^{n}\right]$ is an interval output vector of the $j t h$, which is the average of the consequent type-2 fuzzy set representing the class that input features are assigned to.

Assume the input vector is $x=\left(x_{1}, x_{2}, \cdots, x_{I}\right)$. The operation scheme of type-2 Fuzzy Classifier involve the following steps:

1) Compute the membership of $x_{i}$ on each $\tilde{A}_{i}^{n} \cdot\left[\bar{u}_{\tilde{A}_{i}^{n}}\left(x_{i}\right), \underline{u}_{\tilde{A}_{i}^{n}}\left(x_{i}\right)\right], i=1, \cdots I n=1, \cdots N$

2) Compute the firing interval of the nth rule, $F^{n}(\boldsymbol{x})$ :

$F^{n}(x)=\left[\bar{u}_{\tilde{A}_{i}^{n}}\left(x_{i}\right) \times \cdots \times \bar{u}_{\tilde{A}_{i}^{n}}\left(x_{I}\right), \underline{u}_{\hat{A}_{i}^{n}}\left(x_{i}\right) \times \cdots \times \underline{u}_{\tilde{A}_{i}^{n}}\left(x_{I}\right)\right] \equiv\left[\bar{f}^{n}, \underline{f}^{n}\right]$.

3) Perform type-reduction to combine $F^{n}(x)$ and the corresponding rule consequents. There are many such methods. The most commonly used one is the center-of-sets(COS) type-reducer: 
$Y_{\cos }(\boldsymbol{x})=\underset{\substack{f^{n} \in F^{n}(\boldsymbol{x}) \\ y^{n} \in Y^{n}}}{\sum_{n=1}^{N} f^{n} y^{n}}=\left[y_{l}, y_{r}\right]$.

It has been shown that

$y_{l}=\min _{k \in[1, N-1]} \frac{\sum_{n=1}^{k} \bar{f}^{n} \underline{y}^{n}+\sum_{n=k+1}^{N} \underline{f}^{n} \underline{y}^{n}}{\sum_{n=1}^{k} \bar{f}^{n}+\sum_{n=k+1}^{N} \underline{f}^{n}} \equiv \frac{\sum_{n=1}^{L} \bar{f}^{n} \underline{y}^{n}+\sum_{n=L+1}^{L} \underline{f}^{n} \underline{y}^{n}}{\sum_{n=1}^{L} \bar{f}^{n}+\sum_{n=L+1}^{L} \underline{f}^{n}}$.

$y_{r}=\max _{k \in[1, N-1]} \frac{\sum_{n=1}^{k} \underline{f}^{n} \bar{y}^{n}+\sum_{n=k+1}^{N} \bar{f}^{n} \bar{y}^{n}}{\sum_{n=1}^{k} \underline{f}^{n}+\sum_{n=k+1}^{N} \bar{f}^{n}} \equiv \frac{\sum_{n=1}^{R} \underline{f}^{n} \bar{y}^{n}+\sum_{n=R+1}^{R} \bar{f}^{n} \bar{y}^{n}}{\sum_{n=1}^{R} \underline{f}^{n}+\sum_{n=R+1}^{R} \bar{f}^{n}}$.

where the switch points $\mathrm{L}$ and $\mathrm{R}$ are determined by

$\underline{y}^{L} \leq y_{l} \leq \underline{y}^{L+1}$.

$\bar{y}^{R} \leq y_{r} \leq \bar{y}^{R+1}$.

and $\left\{\underline{y}^{n}\right\}$ and $\left\{\bar{y}^{n}\right\}$ have been sorted in ascending order, respectively. $y_{l}$ and $y_{r}$ can be computed

efficiently using the Karnik-Mendel (KM) algorithms $[15,16]$. To compute $\mathrm{y}_{1}$ and $\mathrm{y}_{\mathrm{r}}$, the steps are

a) Sort $y_{n}(n=1,2, \cdots, N)$ in increasing order and call the sorted $y^{n}$ by the same name, but now $y^{1} \leq$ $y^{2} \leq \cdots \leq y^{N}$. Match the weights $F^{n}(x)$ with their respective $y^{n}$ and renumber them so that their index corresponds to the renumbered $y^{n}$.

b) Compute $y_{l}$ as $y=\frac{\sum_{n=1}^{N} \underline{\underline{y}}^{n} f^{n}}{\sum_{n=1}^{N} f^{n}}$ by initially setting $f^{n}=\frac{f^{n}+\bar{f}^{n}}{2}$ for $n=1,2, \cdots, N$

c) $\quad$ Find $L(1 \leq L \leq N-1)$ such that $\underline{y}^{L} \leq y \leq \underline{y}^{L+1}$

d) Set $f^{n}=\left\{\begin{array}{ll}\bar{f}^{n} & n \leq k \\ f^{n} & n>k\end{array}\right.$ and compute $y^{\prime}=\frac{\sum_{n=1}^{N} \underline{y}^{n} f^{n}}{\sum_{n=1}^{N} f^{n}}$

e) Check if $y^{\prime}=y$. If yes, stop and $y_{l}=y$ and $L=k$. If no, go to Step f.

f) Set $y=y^{\prime}$ and go to Step 3 .

The procedure for computing $y_{r}$ is very similar to the one for $y_{l}$. Just replace $y_{r}$ by $y_{l}$ and, in Step 3 find $R(1 \leq R \leq N-1)$ such that $\underline{y}^{R} \leq y \leq \underline{y}^{R+1}$.

4) Compute the defuzzified output as

$$
y=\frac{y_{l}+y_{r}}{2} .
$$

The operation scheme of designed type-2 Fuzzy Classifier has been shown in Fig. 3, briefly.

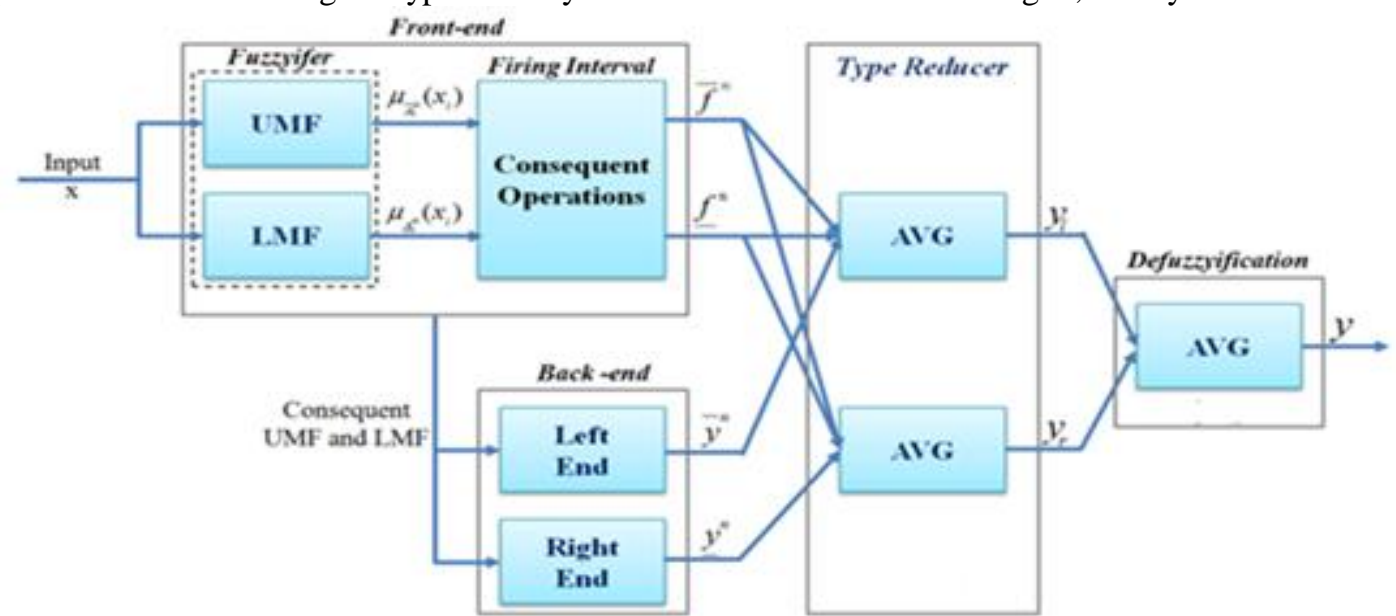

Figure 3. The operation scheme of design of type-2 Fuzzy Classifier using uncertainty UMF and LMF bounds technique.

Type-2 Fuzzy Classifier has been set up, its FOU by taking intersections of all upper and lower memberships for all features by our heuristic method. If we choose the min operation as intersection, the FOU can be expressed as [19]

$\bigcup_{\forall x \in X}[\underline{u}(x), \bar{u}(x)]=\bigcup_{\forall x \in X}\left\{\min _{i}\left[\underline{u}_{i}\left(x_{i}\right)\right], \min _{i}\left[\bar{u}_{i}\left(x_{i}\right)\right]\right\}$ 
where, $\bar{u}(x)$ and $\underline{u}(x)$ are the minimum UMF and LMF among all UMFs and LMFs for all features, respectively. Figure. 4 shows the example of MF of five types emitter RF (low limit) for some data sets obtained from the ABC Group of EID Databases, which is the sample and each of five clusters contains 150 data.

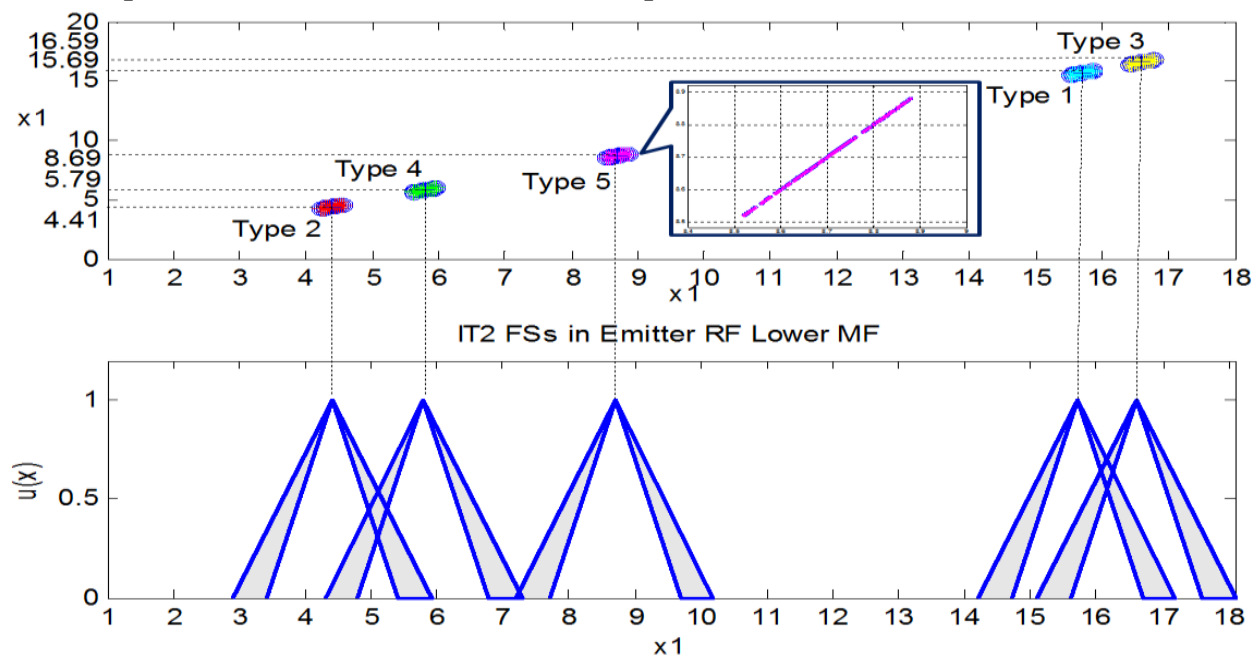

Figure 4. RF (lower limit) for the MFs.

\section{EXPERIMENTAL RESULTS}

In this section, the experiments are performed for the interval valued input data to demonstrate the classification capability of the type-2 Fuzzy Classifier.

The ELINT repository used for this study contains three parameters: The pulse repletion interval (PRI) is in the range of $1.0 \mu \mathrm{s} \sim 10.0 \mu \mathrm{s}$, the pulse width (PW) is in the range of $0.1 \mu \mathrm{s} \sim 10.0 \mu \mathrm{s}$, and the radio frequency of the carrier wave (RF) is in the range of $2.0 \mathrm{GHz} \sim 18.0 \mathrm{GHz}$.

There were a five types radar emitters of experiments carried out in this research reported here in order to examine the capabilities of the proposed type-2 Fuzzy Classifier. Its performance in terms of the error deviation level (EDL) was employer in this section. In this experiment, all data are consisted of 150 samples containing five emitter types from the emitter samples repository of ABC Group. To perform the testing at different levels of additive noise, the error deviation level defined as

$\operatorname{EDL}_{\mathrm{i}}(\%)=\frac{\xi_{\mathrm{pi}}}{\mathrm{x}_{\mathrm{pi}}} \times 100 \%$

where $\mathrm{x}_{\mathrm{pi}}$ is the data without noise and $\xi_{\mathrm{pi}}$ is random noise.

For the interval-value noisy samples is $\mathrm{x}_{\mathrm{p}}=\left(\mathrm{x}_{\mathrm{p} 1}, \mathrm{x}_{\mathrm{p} 2}, \mathrm{x}_{\mathrm{p} 3}\right)$, briefly, $\mathrm{x}_{\mathrm{pi}}=\left[\mathrm{x}_{\mathrm{pi}}^{\mathrm{L}}, \mathrm{x}_{\mathrm{pi}}^{\mathrm{U}}\right]$ superscript $\mathrm{L}$ denoted as lower limit and superscript $\mathrm{U}$ as upper limit. $\xi_{\mathrm{pi}}=\left[\xi_{\mathrm{pi}}^{\mathrm{L}}, \xi_{\mathrm{pi}}^{\mathrm{U}}\right]$ is the random noise corresponding to $\mathrm{x}_{\mathrm{pi}}$, then the noisy testing sample will be $\mathrm{x}_{\mathrm{pi}}=\left[\mathrm{x}_{\mathrm{pi}}^{\mathrm{L}} \pm \xi_{\mathrm{pi}}^{\mathrm{L}}, \mathrm{x}_{\mathrm{pi}}^{\mathrm{U}} \pm \xi_{\mathrm{pi}}^{\mathrm{U}}\right]$. Figure. 5 shows an example of triangular Type2 Fuzzy Classifier MFs that are generated from the sample data set of Table 1.
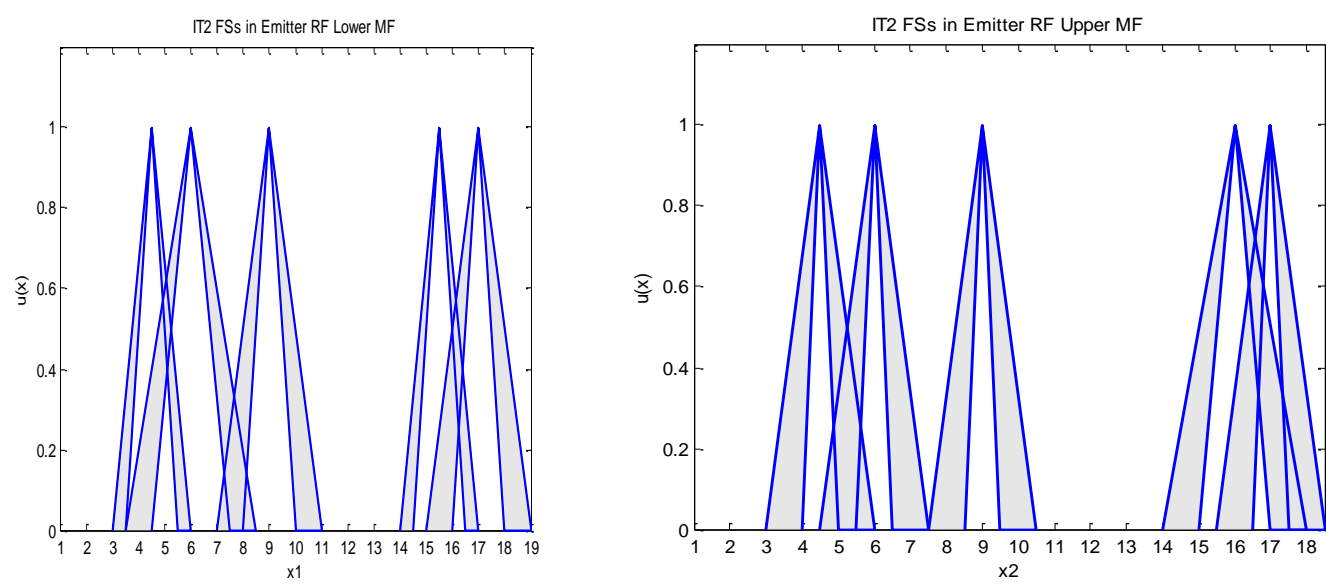

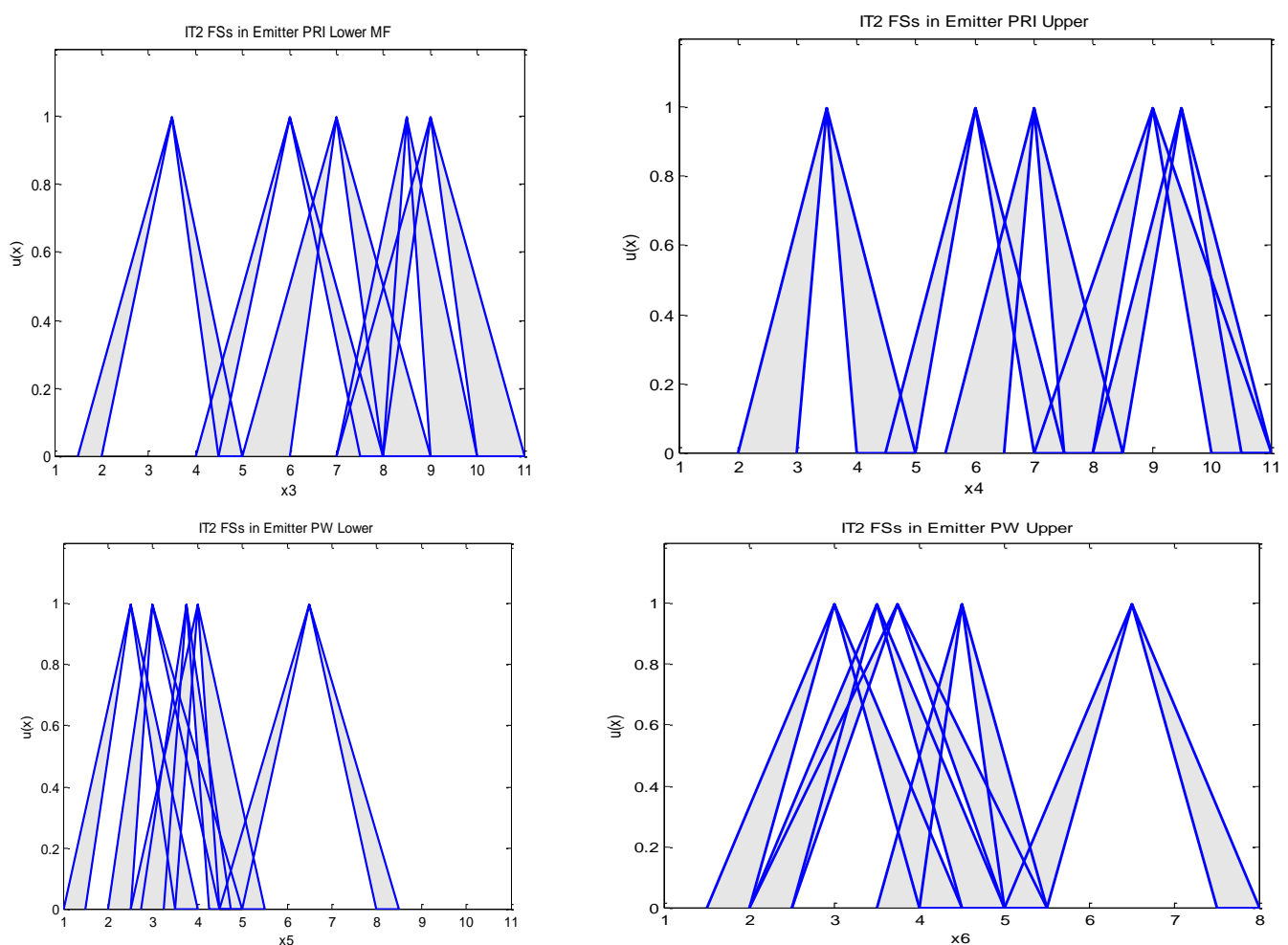

Figure 5. Type-2 Fuzzy Classifier for antecedent MFs' UMF/ LMF

$\mathrm{n}$ this experiment, jitter is normally described in terms of its probability density function (PDF) on lower limit( $\mathrm{U}$ ) edge and upper limit (U) edge. Wiley [22] states that the measurement jitter due to noise is white Gaussian, then the corresponding interval $\left[\mathrm{x}_{\mathrm{pi}}^{\mathrm{L}}, \mathrm{x}_{\mathrm{pi}}^{\mathrm{U}}\right]$ is produced as

$\mathrm{x}_{\mathrm{pi}}^{\mathrm{L}}=\mathrm{x}_{\mathrm{pi}}-\operatorname{rand}\left[\left(\mathrm{x}_{\mathrm{pi}}-\operatorname{MinX}\right) \cdot \mathrm{r}\right]$

$\mathrm{x}_{\mathrm{pi}}^{\mathrm{U}}=\mathrm{x}_{\mathrm{pi}}+\operatorname{rand}\left[\left(\mathrm{x}_{\mathrm{pi}}-\operatorname{MinX}\right) \cdot \mathrm{r}\right]$

where $\operatorname{rand}(\mathrm{x}) \in[0, \mathrm{x}]$ is the random function, MinX is the minimum value of all $\mathrm{x}_{\mathrm{pi}}$ in the whole sequence, and $r \in[0, x]$ is a real number, which is the relative random ratio $(r=0.2$ in this experiment).

To perform the testing at different levels of introduce the jitter variation to produce testing samples. In this experiment, the testing samples with different EDLs (from 0 to 20\%) are presented to the Type-2 Fuzzy Classifier for performance testing. The testing results which also gained through 100 Monte Carlo simulations are listed in Table 2.

From Table 2, type-2 Fuzzy Classsifier perform achieves an average EDL rate(from 0 to $20 \%$ ) of $87.5 \%$. This explained type-2 Fuzzy Classifier advantage and effectively reduces the effects of uncertainties in radar emitter interval-value signals with and jitter variations.

Table 2 Average EDL performance of type-2 Fuzzy Classifier testing samples with jitter variation

\begin{tabular}{|c|c|c|c|c|c|c|c|c|c|c|c|}
\hline \multirow[b]{2}{*}{ EDL } & \multicolumn{11}{|c|}{ Type-2 Fuzzy Classifier Performance } \\
\hline & 20 & 18 & 16 & 14 & 12 & 10 & 8 & 6 & 4 & 2 & 0 \\
\hline $\begin{array}{l}\text { TotalAverage } \\
\text { Accuracy }(0)\end{array}$ & 70.5 & 75.7 & 79.2 & 83.7 & 87.5 & 89.6 & 909 & 92.2 & 94.5 & 987 & 100 \\
\hline
\end{tabular}




\section{CONCLUSION}

This paper discuss the type-2 Fuzzy Classifier, analyzes the insensitive to jitter of the EDI classification problem. The type-2 Fuzzy Classifier has shown its superior capabilities to handle the uncertainty. The robustness to noise is here of special importance. Monte Carlo simulation results show that the type-2 Fuzzy Classifier not only achieves better identification capability, but also obtains better adaptability to jitter. With these results achieved in this paper, the proposed type-2 Fuzzy Classifier may be embedded into ELINT system and enhance military applications (such as reconnaissance and threat reaction). In addition, as part of future work it is intended to explore the ways that uncertainty bounds of the classifier's output can be effectively exploited with a view to improving the performance of the classifier. Only the central point of the output interval has been utilized so far.

\section{ACKNOWLEDGMENTS}

This research work was sponsored by the National Science Council, R.O.C., under project number NSC1022623-E-155-002-D.

\section{REFERENCES}

[1] J. Roe, S. Cussons, and A. Feltham: Knowledge-based signal processing for radar ESM systems, IEE Proceedings F, Radar and Signal Processing, 137(5), 1990, 293-301

[2] Y.J. Ryoo, K.H. Song, and W.W. Kim, Recognition of PRI modulation types of radar signals using the autocorrelation', IEICE Trans. Commun., E90-B(5),2007, 1290-1294

[3] A. K. Chang, J.O., Ryu and Y. Kim, Comparison of methods to estimate individual tree attributes using color aerial photographs and LiDAR data', WSEAS Trans. Signal Process., 4(1), 2008, 21-27

[4] J. Liu, J. Lee, L. Li, Z.Q. Luo, and K. Wong ,Online clustering algorithms for radar emitter classification, IEEE Trans. Pattern Anal. Mach. Intell., 27(8), 2005,1185-1196

[5] S.A. Hassan, A.I. Bhatti, and A. Latif, Emitter recognition using fuzzy inference system, IEEE Int. Conf. Emerging Technologies, 2005, 204-208

[6] E. Granger, M. Rubin, S. Grossberg, and P. Lavoie, A what-and-where fusion neural network for recognition and tracking of multiple radar emitters, Neural Networks, 14(3),2001, 325-344

[7] H. Rong, W. Jin, and C. Zhang, Application of support vector machines to pulse repetition interval modulation recognition, In Proceedings of 6th international conference on ITS telecommunications, 2006, $1187-1190$

[8] S.J. Thakur, K. Sing, D.K. Basu, and M. Nasipuri, Face recognition using principal component analysis and RBF neural networks, Proceedings of 1st International Conference on Emerging Trends in Engineering and Technology, 2008, 695-700

[9] S. Rabinovich, Measurement Errors: Theory and Practice, (American Institute of Physics, New York, 2005)

[10] A. Niewiadomski andM. Bartyzel, Elements of type-2 semantics in summarizing databases', Lecture Notes in Artificial Intelligence, vol. 4029, 2006, 278-287

[11] J.M. Mendel and H. Wu, New results about the centroid of an interval type-2 fuzzy set, including the centroid of a fuzzy granule, Information Sciences, 177,2007, 360-377.

[12] L.A. Zadeh, The concept of a linguistic variable and its application to approximate reasoning, Information Sciences, 8, 1975, 199-249

[13] H.B. Mitchell, Pattern recognition using type-II fuzzy sets, Information Sciences, 170, 2005, 409-418.

[14] X. Chen, Y. Li, R. Harrison andY.Q. Zhang, Type-2 fuzzy logic-based classifier fusion for support vector machines, Applied Soft Computing, 8, 2008, 1222-1231.

[15] N.N. Karnik and J.M. Mendel, Centroid of a type-2 fuzzy set', Information Sciences, 132, 2001, 195-220.

[16] D. Wu and J. M. Mendel, Enhanced Karnik-Mendel Algorithms, IEEE Trans. on Fuzzy Systems, 17(4), 2009, 923-934.

[17] C.H. Rhee, Uncertain fuzzy clustering: insights and recommendations, IEEE Computation. Intelligent Mag., 2007, 44-56.

[18] C.F. Hwang and C.H. Rhee, Uncertain fuzzy clustering: interval type-2 fuzzy approach to C-means, IEEE Trans. Fuzzy System, 15(1),2007, 107-120.

[19] B.I. Choi and C.H. Rhee, Interval type-2 fuzzy membership function generation methods for pattern recognition, Information Sciences, 179, 2009, 2102-2122.

[20] R.G. Wiley, The Analysis of Radar Signals', (Artech House, Norwood, MA, 2nd ed, 1982) 